\title{
Article \\ Mirror and Circular Symmetry of Autofocusing Beams
}

\author{
Svetlana N. Khonina ${ }^{1,2}$ iD
}

1 IPSI RAS-Branch of the FSRC “Crystallography and Photonics” RAS, Molodogvardeyskaya 151, 443001 Samara, Russia; khonina@ipsiras.ru

2 Department of Technical Cybernetics, Samara National Research University, Moskovskoye Shosse 34, 443086 Samara, Russia

Citation: Khonina, S.N. Mirror and Circular Symmetry of Autofocusing Beams. Symmetry 2021, 13, 1794. https://doi.org/10.3390/ sym13101794

Academic Editor: Christophe Humbert

Received: 22 August 2021

Accepted: 23 September 2021

Published: 26 September 2021

Publisher's Note: MDPI stays neutral with regard to jurisdictional claims in published maps and institutional affiliations.

Copyright: (C) 2021 by the author. Licensee MDPI, Basel, Switzerland. This article is an open access article distributed under the terms and conditions of the Creative Commons Attribution (CC BY) license (https:/ / creativecommons.org/licenses/by/ $4.0 /)$.

\begin{abstract}
This article demonstrates the crucial importance of the symmetrization method for the formation of autofocusing beams. It is possible to impart autofocusing properties to rather arbitrary distributions, for example, truncated and inverted classical modes (such as Hermite-Gaussian, Laguerre-Gaussian, and Bessel modes) or shift the fundamental Gaussian beam by inserting mirror or circular symmetry. The most convenient for controlling autofocusing characteristics is the truncated sinus function with a power-law argument dependence. In this case, superlinear chirp beams (with power $q>2$ ) exhibit sudden and more abrupt autofocusing than sublinear chirp beams (with power $1<q<2$ ). Comparison of the different beams' propagation is performed using fractional Fourier transform, which allows obtaining the field distribution in any paraxial region (both in the Fresnel and Fraunhofer diffraction regions). The obtained results expand the capabilities of structured beams in various applications in optics and photonics.
\end{abstract}

Keywords: autofocusing beams; accelerating beams; mirror and circular symmetry; laser modes; fractional Fourier transform

\section{Introduction}

A laser beam propagating in a linear medium can exhibit properties similar to the action of a lens if a phase gradient is present in the transverse distribution of the beam. This phenomenon is commonly called autofocusing. Circular Airy beams [1-4] are characterized by the property of abrupt autofocusing. This is a consequence of the accelerating property of 1D Airy beams [5,6], which propagate along a curved path according to the parabolic law. The peculiarity of abrupt autofocusing is that the beam, at first, approximately retains its original distribution and then abruptly focuses. Beams with autofocusing properties are useful for optical manipulation [7,8], multiphoton polymerization [9], nonlinear effects [10], polarization conversion [11], and sharp focusing [12]. Since there is such a demand in various applications, researchers have focused on the study of other beams with autofocusing properties, for example, Pearcey beams [13,14], aberration beams [15], as well as symmetric [16-18] and generalized [19,20] Airy beams.

Note that accelerating beams have an autofocusing property when a certain symmetry is introduced. In particular, the "specular" Airy beams proposed in [16] are similar to the Hermite-Gaussian (HG) modes and do not have autofocusing characteristics. During propagation, the specular Airy beams demonstrate a symmetrical acceleration in opposite directions, i.e., divergence is proportional to the square of the distance traveled. In addition, depending on the type of circular symmetry, one can obtain autofocusing beams or structurally stable beams similar to the Laguerre-Gaussian (LG) modes.

In this work, we studied in detail the influence of the symmetry properties on the characteristics of the propagation trajectory of various beams, including those previously not considered, for example, half-HG beams, truncated Bessel beams, and inverted LG beams. For numerical simulation, the fractional Fourier transform (FrFT) [21-23] was used, which describes the propagation of beams in lens systems [24,25] as well as in media with a gradient refractive index $[26,27]$. 
Beams with controlled propagation properties have the potential for a wide range of applications in optics and photonics.

\section{Acceleration of Asymmetric Beams}

In this section, for numerical simulation of beam propagation, we used the 1D FrFT [21-23]:

$$
\begin{gathered}
G(u, z)=\sqrt{-\frac{i k}{2 \pi f \sin (\tau z)}} \exp \left\{\frac{i k u^{2} \cos (\tau z)}{2 f \sin (\tau z)}\right\} \times \\
\int_{-D / 2}^{D / 2} g(x) \exp \left[\frac{i k x^{2} \cos (\tau z)}{2 f \sin (\tau z)}-\frac{i k x u}{f \sin (\tau z)}\right] \mathrm{d} x,
\end{gathered}
$$

where $k=2 \pi / \lambda, \lambda$ is the light wavelength, $f$ is the focal length of a lens, $\tau=\pi /(2 f), z$ is the distance from the input plane, $D$ is the field size in the input plane, and $x$ and $u$ are transverse coordinates in the input and output plane, respectively.

The propagation integral operator (1) allows the field distribution to be obtained in any paraxial region, both in the Fresnel and Fraunhofer diffraction regions.

It is well-known that the Airy beams have a remarkable property of acceleration in free space, which means a deviation from the straight trajectory according to parabolic law $[5,6,28,29]$. However, other beams with an asymmetric distribution can have similar properties, for example, cosh-Airy beams [30], half-Pearcey beams [31], and 1D Pearcey beams [32].

Let us consider the truncated Airy (tAi) functions [16,18]:

$$
g_{t A i}(x)=\operatorname{Ai}\left(\frac{x-x_{0}}{c}\right) \operatorname{rect}\left(\frac{x}{b-a}\right),
$$

where $\operatorname{Ai}(x)$ is the Airy function [33], $x_{0}$ is the shift parameter, $c$ is the scale parameter, and $\operatorname{rect}(x)$ is the truncation function:

$$
\operatorname{rect}\left(\frac{x}{b-a}\right)=\left\{\begin{array}{c}
1, \quad x \in[a, b] \\
0, \text { else. }
\end{array}\right.
$$

The Airy beam (2) has a spatial asymmetry (Figure 1a).

In $[16,18]$, a comparison between Airy mirror beams and HG modes was made; therefore, we compared the propagation of the beam (2) and the beam representing half of the HG mode:

$$
g_{h H G}(x)=\exp \left(-\frac{\left(x-x_{0}\right)^{2}}{2 \sigma^{2}}\right) H_{n}\left(\frac{x-x_{0}}{\sigma}\right) \operatorname{rect}\left(\frac{x}{b-a}\right),
$$

where $H_{n}(x)$ is the Hermite polynomial of the $n$th order [33] and $\sigma$ is the Gaussian parameter. Note, for $n=0$, expression (4) corresponds to the shifted fundamental Gaussian beam.

In addition, let us consider truncated Bessel (tBs) functions:

$$
g_{t B S}(x)=J_{n}\left(\frac{x-x_{0}}{c}\right) \operatorname{rect}\left(\frac{x}{b-a}\right),
$$

where $J_{n}(x)$ is the Bessel function of the $n$th order [33].

The parameters of functions (2), (4) and (5) can be chosen so that they are similar in structure. In particular, Figure 1a shows the input distributions (amplitudes of real functions) fitted so that the number of zeros and the position of the maximum values coincide.

To compare the accelerating features of the different beams, we used operator (1) with the calculation parameters: $\lambda=633 \mathrm{~nm}, D=2 \mathrm{~mm}$, and $f=150 \mathrm{~mm}$. The displacement of the beam $\Delta$ was estimated as the difference between the positions of the maximum value at the input plane and at the distance $z=f / 2=75 \mathrm{~mm}$. As seen from the simulation 
results (Figure $1 \mathrm{~b}$ ), the displacement $\Delta$ for the conventional Gaussian beam is very small and corresponds to a linear propagation trajectory. For the rest of the beams, acceleration during propagation was observed. Moreover, the displacement $\Delta$ for the half-HG beam is slightly larger than that for the Airy beam and even larger for the tBs beam.

Note that the spatial spectrum (Figure $1 \mathrm{c}$, distribution in the focal plane $z=f$ ) of the half-HG beam and the tBs beam is more compact than the tAi beam. This means that the tAi beam has more energy at high frequencies and, therefore, the tAi beam itself contains more frequent oscillations in the peripheral part than the other two beams. This can have both a positive meaning (for example, a significant variation in the size of light spots provided) and a negative effect (for example, faster attenuation or distortion of the beam in paraxial optical systems).

(a)

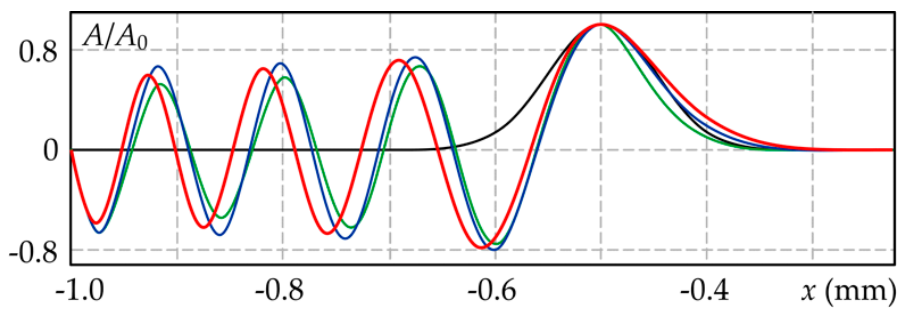

(b)

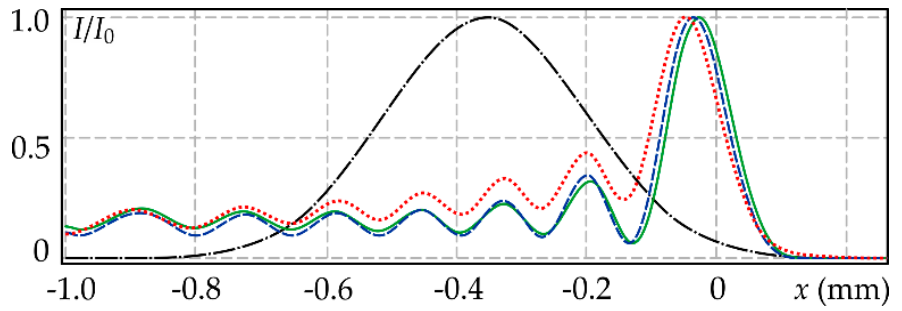

(c)

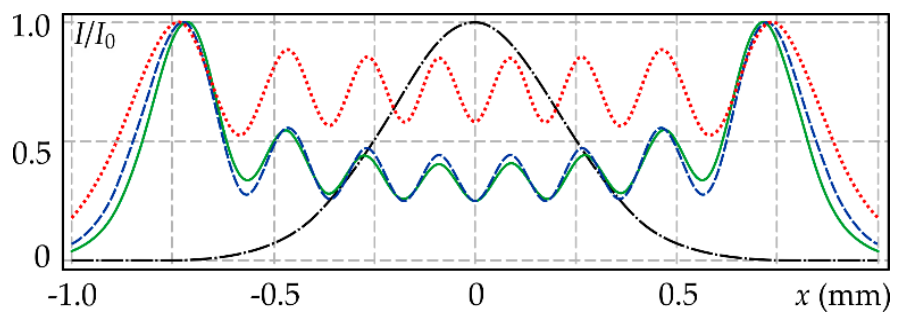

Figure 1. Comparison of accelerating features of different asymmetric beams: Gaussian beam of Equation (4) ( $n=0$, black color), tAi beam of Equation (2) (red color), half-HG beam of Equation (4) ( $n=15$, blue color), and tBs beam of Equation (5) ( $n=30$, green color): (a) input distribution (amplitudes of real functions); (b) distribution of normalized intensity at the distance $z=f / 2$ (displacement is $\Delta=0.148 \mathrm{~mm}$ for the Gaussian beam, $\Delta=0.452 \mathrm{~mm}$ for the tAi beam, $\Delta=0.468 \mathrm{~mm}$ for the half-HG beam, and $\Delta=0.476 \mathrm{~mm}$ for the tBs beam); (c) focal plane normalized intensity distribution in the focal plane $(z=f)$.

Thus, it is possible to generate beams that are displaced more than Airy beams, but this does not mean that the trajectory is accelerating more. It is rather difficult to define the analytical form of the trajectory of investigated beams; however, complicated functions can be approximated by simpler functions. For example, in papers $[2,4,12,34]$, autofocusing beams are considered in the form of sinus functions with an arbitrary powerlaw dependence on the radius. Such beams are convenient for analysis and can be used to approximate various functions.

For the convenience of this analysis, we also considered beams described by a simpler but rather general formula in the form of a truncated sinus function (tSq) with a power-law dependence of the argument: 


$$
g_{t S q}(x)=\sin \left[\left(\frac{x-x_{0}}{c}\right)^{q}\right] \operatorname{rect}\left(\frac{x}{b-a}\right),
$$

where $q$ is a real positive value.

Table 1 shows the results of comparing the propagation of various asymmetric beams (left column) and their counterparts in the form of $\mathrm{tSq}$ beams of Equation (6) (right column). The asymptotic expression for the Airy function is known to be proportional to $\operatorname{Ai}(-x) \simeq\left(\pi^{2} x\right)^{-1 / 4} \sin \left(2 x^{3 / 2} / 3+\pi / 4\right)$ [2], so it is logical to use $q=1.5$ in Equation (6) to approximate the $\mathrm{tAi}$ beam by the $\mathrm{tSq}$ function. However, when taking into account the displacement of the main lobe $\Delta$, the value $q=2$ is more appropriate than $q=1.5$ (Table 1, 1A and 1B). Interestingly, the parameter $q=1.5$ is more suitable for the half-HG beam approximation (Table 1, 2A and 2B). It is expected that the $\mathrm{tBs}$ beam has a better approximation at $q=1$ (Table $1,3 \mathrm{~A}$ and $3 \mathrm{~B})$.

It can be observed from the results shown in Table 1 that with a decrease in the degree of nonlinearity $q$, the displacement of the main lobe $\Delta$ increases. Moreover, an arbitrarily large displacement can be provided by inserting an additional linear phase in the input beam. In particular, let us consider a conventional Gaussian beam with an additional shifting phase:

$$
g_{G \gamma}(x)=\exp \left(-\frac{\left(x-x_{0}\right)^{2}}{2 \sigma^{2}}\right) \exp (i \gamma x),
$$

where $\gamma$ is a real value corresponding to the tilt of the shifting phase.

A simply shifted Gaussian beam (7) can be observed propagating along a linear trajectory toward the center in the focal plane (Table $1,4 \mathrm{~A})$. If we add a linear phase $(\gamma \neq 0)$, then the trajectory will remain linear, but the beam will propagate at a large angle to the optical axis, and it will be shifted in the focal plane.

Thus, the asymmetry and nonlinear dependence in the transverse distribution of beams provide a nonlinear characteristic to the propagation trajectory (acceleration property); however, for simple control of the beam displacement, linear dependences can also be used.

Table 1. Comparison of the asymmetric beams' propagation: graphs of intensity at $z=0$ (blue color) and $z=f / 2$ (red color) and intensity distribution at propagation (the horizontal axis is $z \in[15 \mathrm{~mm}$, $285 \mathrm{~mm}]$, the vertical axis is $x \in[-1 \mathrm{~mm}, 1 \mathrm{~mm}])$.

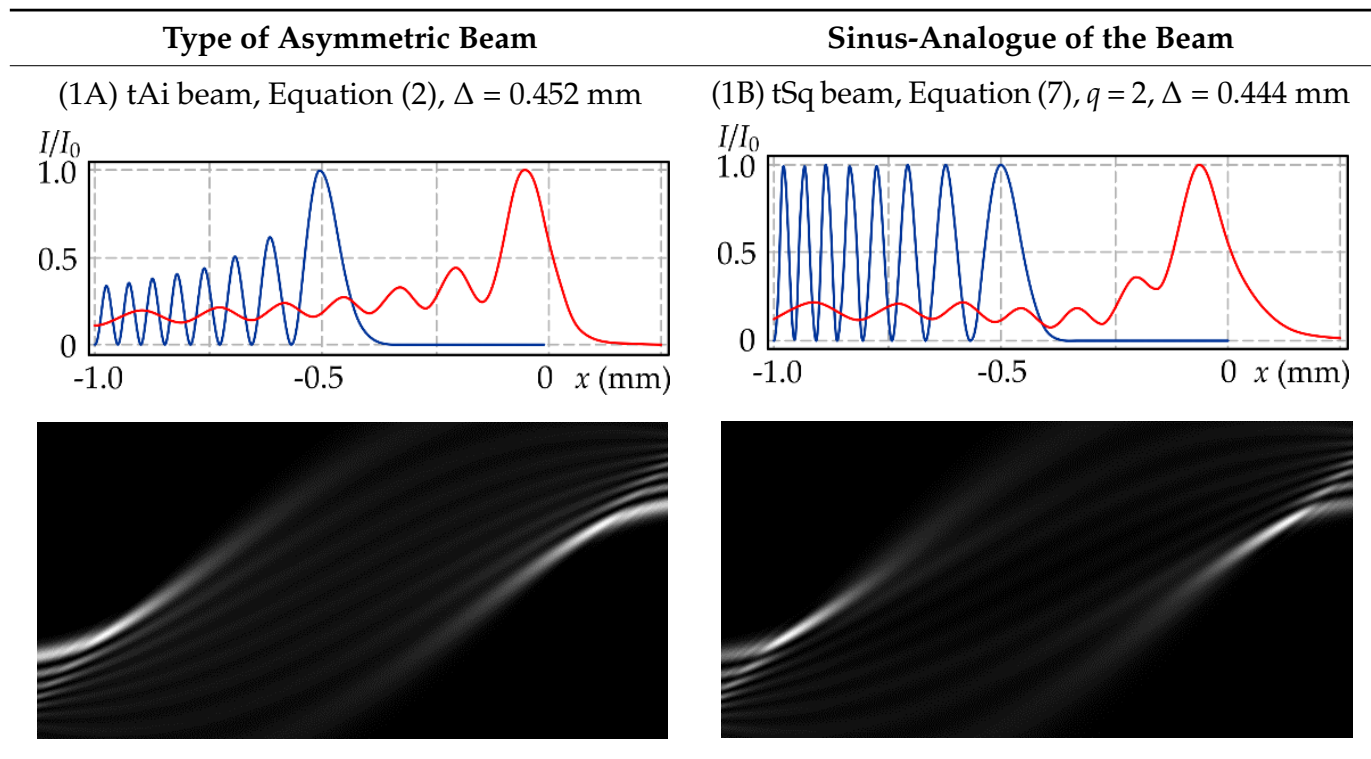


Table 1. Cont.

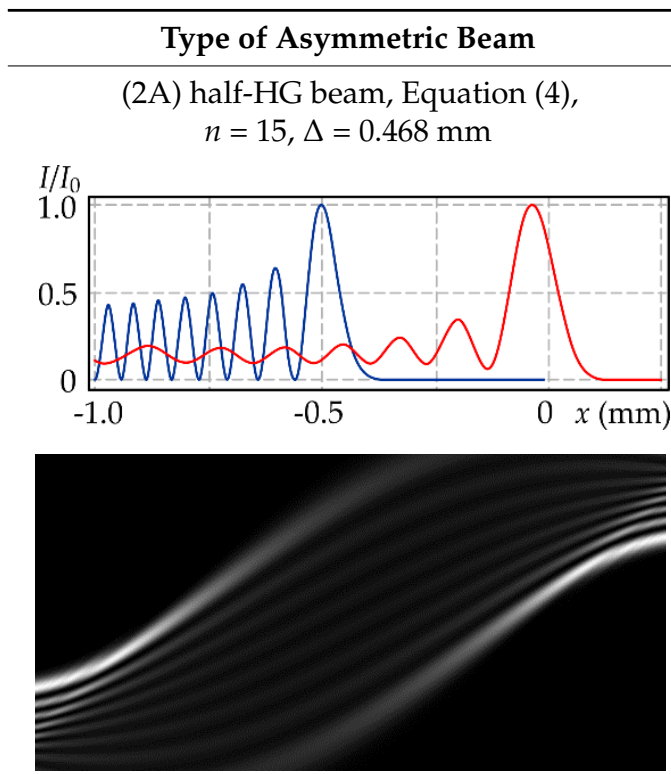

(3A) tBs beam, Equation (5), $n=2, \Delta=0.496 \mathrm{~mm}$

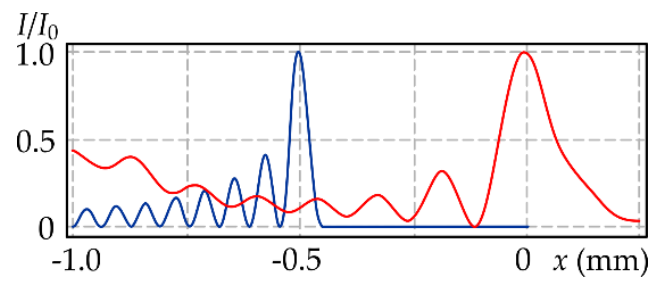

(3B) $\mathrm{tSq}$ beam, Equation (6), $q=1, \Delta=0.488 \mathrm{~mm}$
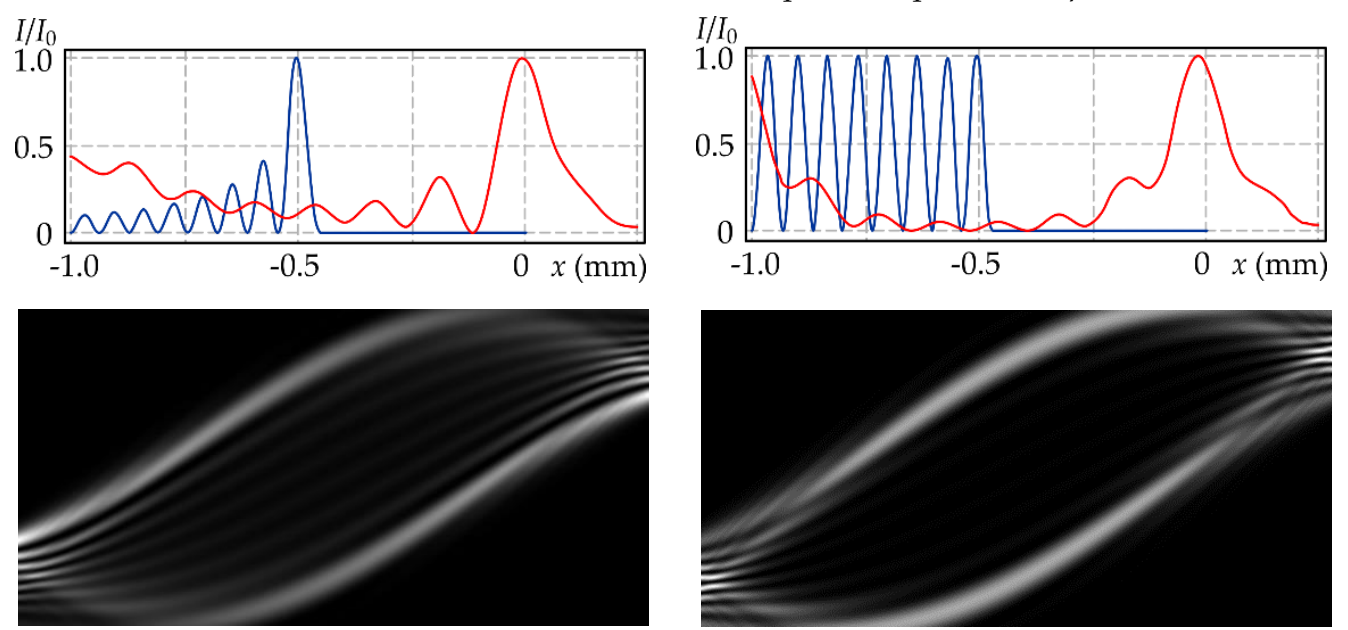

(4A) Gaussian beam, Equation (4), $n=0, \Delta=0.148 \mathrm{~mm}$

(4B) $\mathrm{G} \gamma$ beam, Equation (7), $\gamma=50 \mathrm{~mm}^{-1}, \Delta=0.680 \mathrm{~mm}$
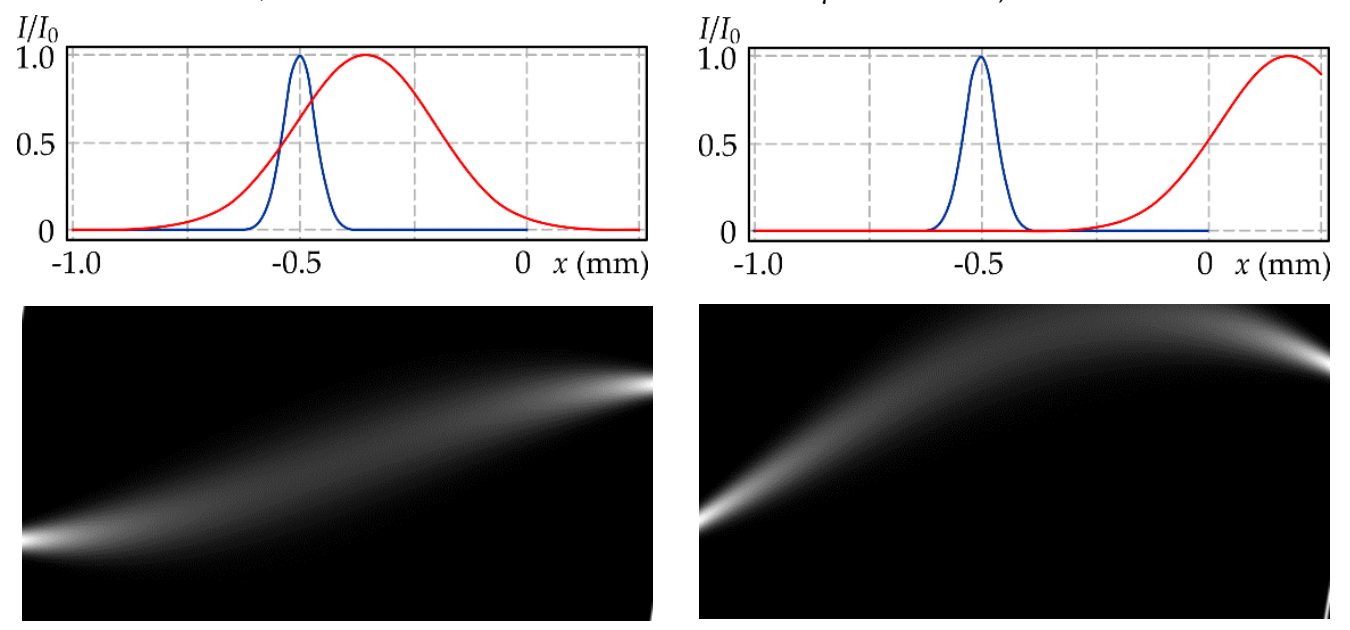


\section{Results}

The asymmetric beams discussed in the previous section can be made symmetrical in various ways. In one case, they will converge and demonstrate the autofocusing property, and in the other case, they will diverge and may maintain structural stability.

\subsection{Beams with a Mirror Symmetry}

Table 2 shows the simulation results (obtained using the operator (1)) for autofocusing mirror beams (position of $z_{\max }$ corresponds to the autofocusing distance) formed from the mirror reflection of asymmetric beams from the previous section:

$$
g_{M}(x)=g(x) \pm g(-x),
$$

where the \pm sign is chosen so that the interference of mirror beams' parts occurs in phase at the autofocusing distance $z_{\max }$. This distance for the investigated beams is determined numerically. However, for the $\mathrm{tSq}$ beams (Equation (6) with $x_{0}=0$ ), the analytical expression of the autofocusing distance is known [20]:

$$
z_{\max }=\frac{2 f}{\pi} \operatorname{arctg}\left(\frac{k c^{2}}{2 f}\right)
$$

Table 2. Comparison of the propagation of mirror beams with autofocusing properties.

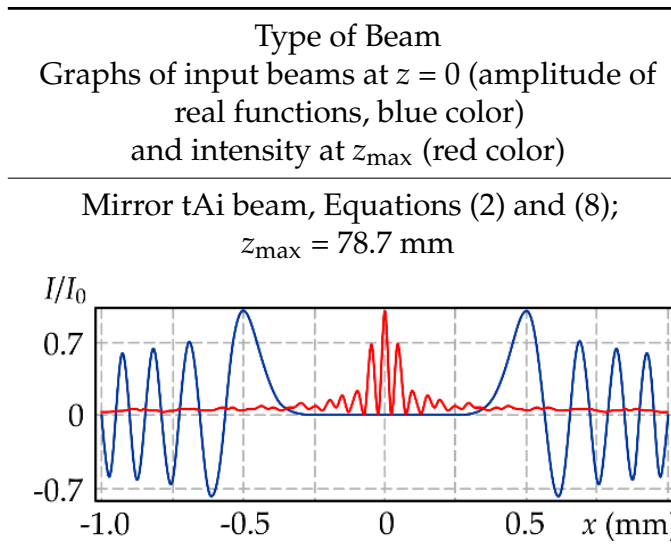

Intensity distribution at propagation (the horizontal axis is $z \in[15 \mathrm{~mm}, 285 \mathrm{~mm}]$, the vertical axis is $x \in[-1 \mathrm{~mm}, 1 \mathrm{~mm}])$

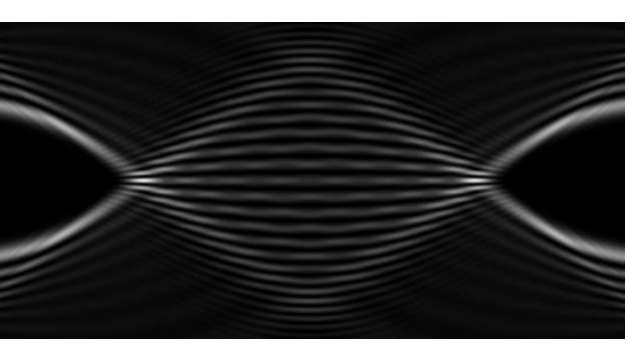

Mirror half-HG beam, Equations (4) and (8), $n=15 ; z_{\max }=77.6 \mathrm{~mm}$
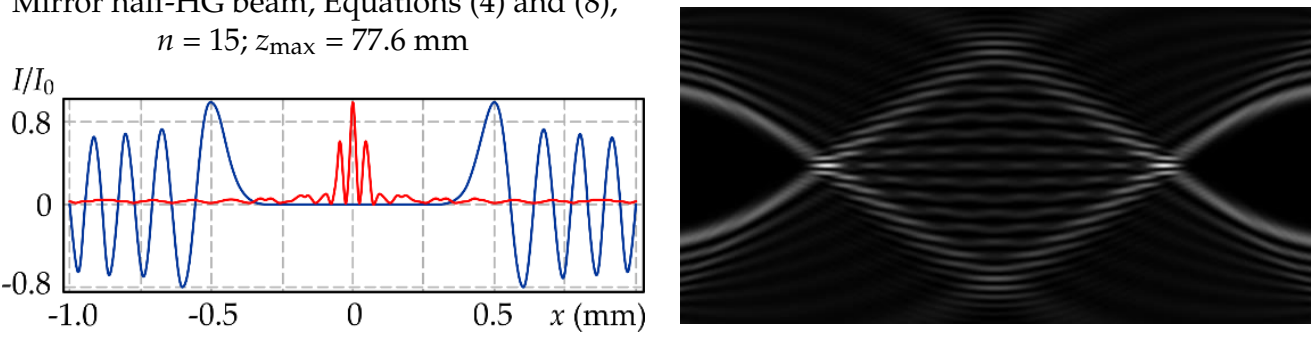

Mirror tSq beam, Equations (6) and (8), $q=1.5$; $z_{\max }=77.7 \mathrm{~mm}$

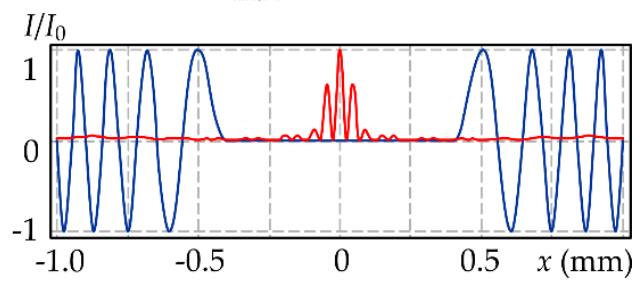

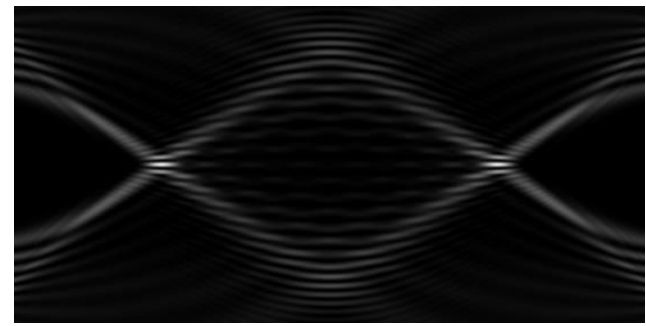


Table 2. Cont.

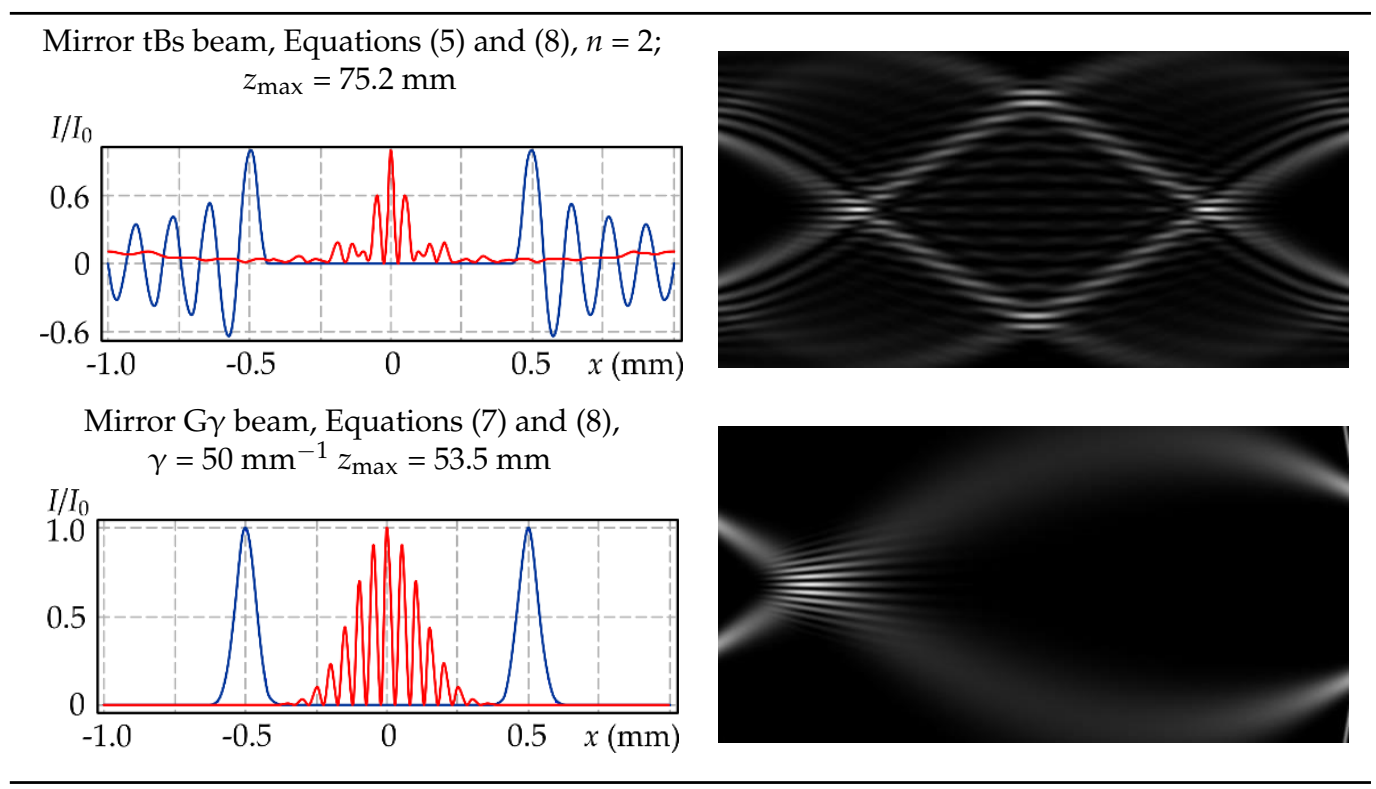

As follows from expression (9), it is possible to generate beams focusing at a certain distance regardless of the nonlinearity of the beam, i.e., degree $q$, only due to variations in parameter $c$. However, the nonlinearity of the trajectory is important if, for example, any beam needs abrupt autofocusing.

As seen from the results shown in Table 2, the autofocusing property can be obtained for various beams if they are provided with mirror symmetry. In this case, the nonlinear transverse beam distribution, for example, as in Airy beams, half-HG beams, or tSq beams (Table 1, lines 1, 2 and 3), provides a nonlinear trajectory and abrupt autofocusing. The linear dependence of the function on the argument (Table 1, lines 4 and 5) corresponds to the linear nature of the trajectory, which is easily controlled by the initial parameters. Different types of autofocusing behavior can be useful in different applications.

Asymmetric beams can be symmetrized in another way, for example, after the preliminary shifting:

$$
g_{S}(x)=g\left(x-x_{S}\right) \pm g\left[-\left(x-x_{S}\right)\right]
$$

where $x_{s}$ is the shift parameter, in general, $x_{s} \neq x_{0}$. The properties of the beams change noticeably depending on the value of $x_{s}$.

Table 3 shows the simulation results (obtained using the operator (1)) for symmetrized beams that diverge, including those that maintain structural stability. In particular, it is seen that the symmetric tAi beam retains its structure quite well (Table 3, line 1), almost similarly to HG mode (Table 3, line 2). Interesting results are also obtained for the symmetric tBs beam (Table 3, line 4) and the symmetric shifted Gaussian beam with a divergent prismatic phase of Equation (7) (Table 3, line 5).

Thus, different symmetrization methods provide different properties of the beams. 
Table 3. Comparison of the propagation of divergent symmetrized beams.

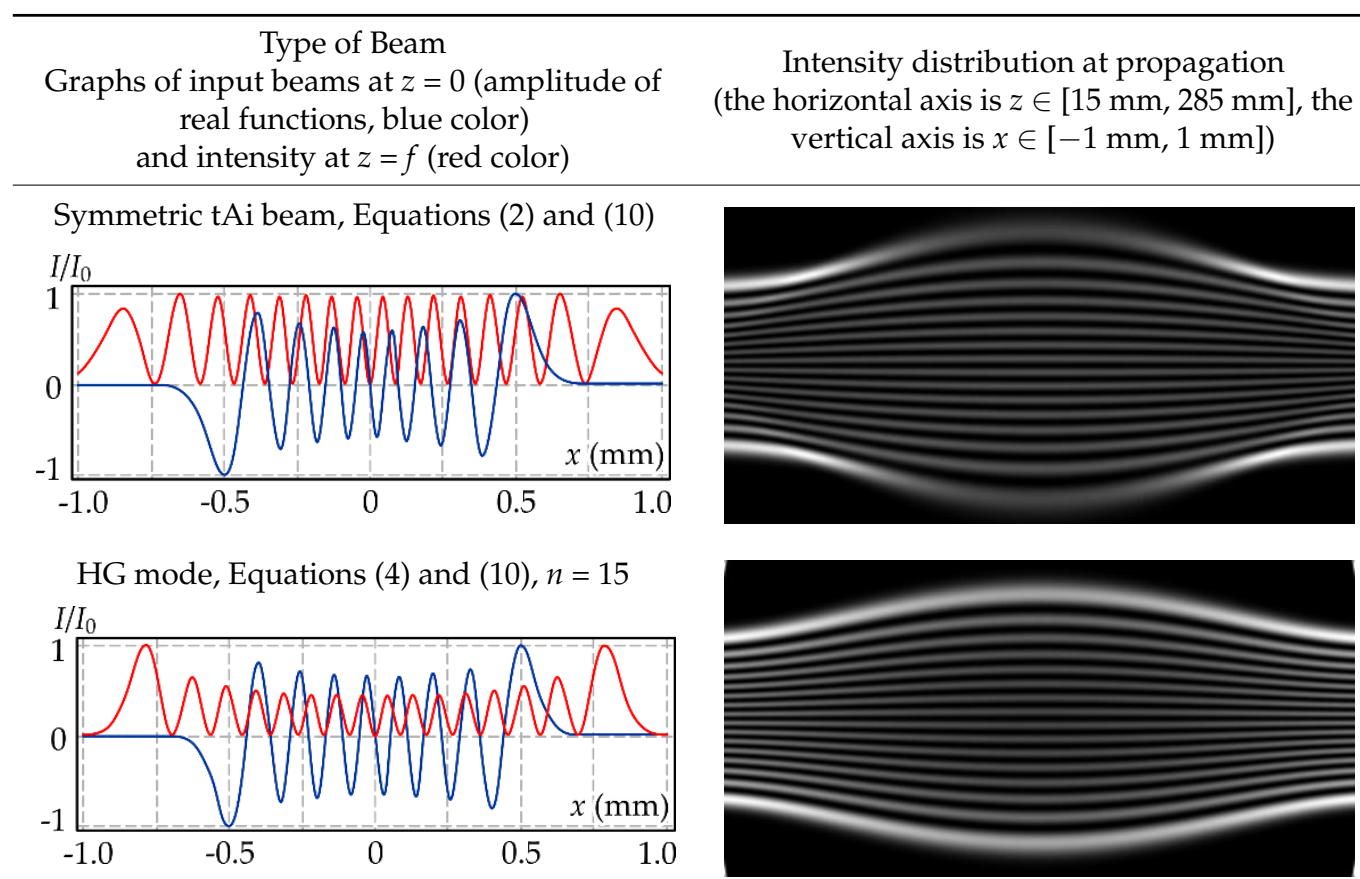

Symmetric tBs beam, Equations (5) and (10),
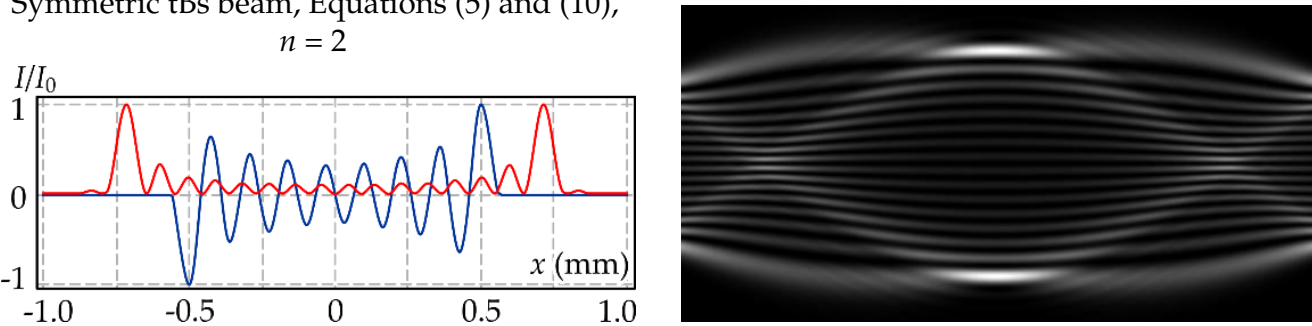

Symmetric $\mathrm{tSq}$ beam, Equations (6) and (10), $q=0.75$
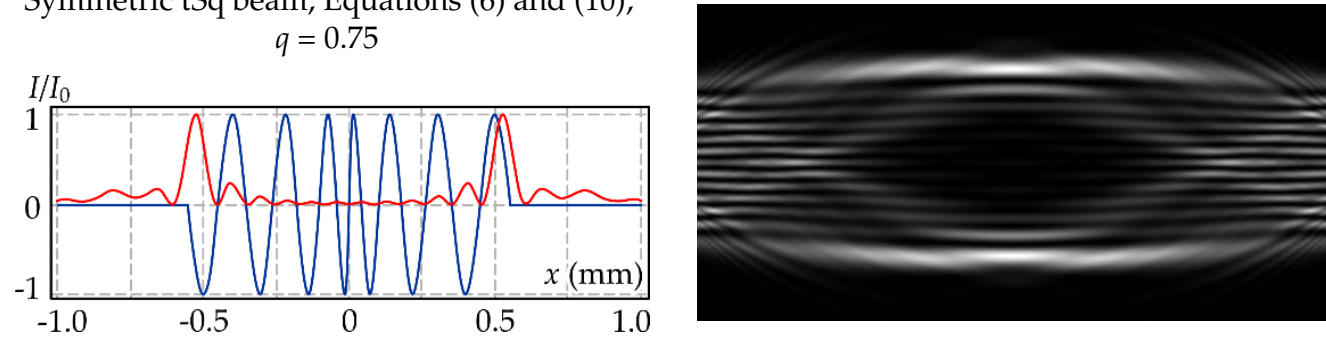

Symmetric $G \gamma$ beam, Equations (7) and (10),

$$
\gamma=45 \mathrm{~mm}^{-1}
$$
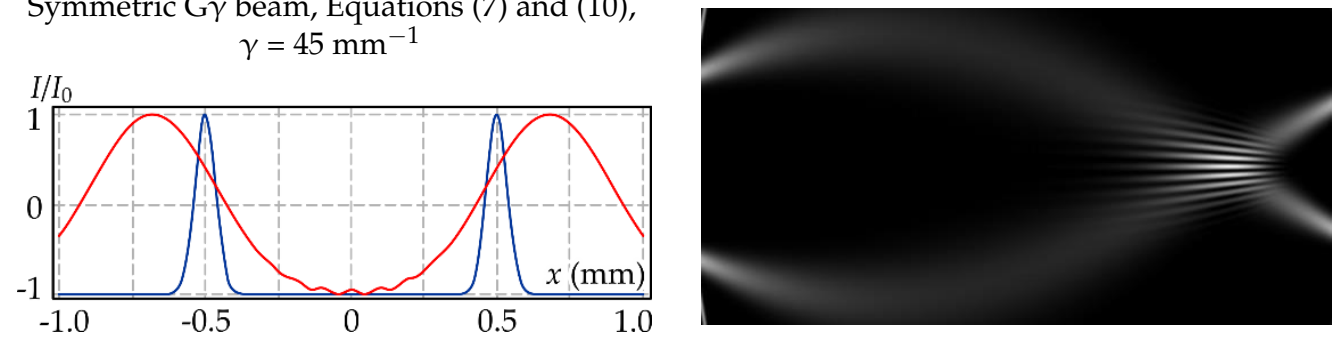

\subsection{Beams with a Circular Symmetry}

Another way to symmetrize asymmetric beams is to insert a circular symmetry:

$$
g_{C}(r)=g\left(r-r_{C}\right)
$$


where $r_{c}$ is the radial shift parameter. By analogy with the previous section, the beam properties change noticeably depending on the $r_{c}$ value.

To simulate the propagation of beams with the circular symmetry, we use the radial FrFT $[25,27]$ :

$$
\begin{aligned}
& G(\rho, z)=-\frac{i k}{f \sin (\tau z)} \exp \left\{\frac{i k \rho^{2} \cos (\tau z)}{2 f \sin (\tau z)}\right\} \times \\
& \int_{0}^{R} g(r) \exp \left[\frac{i k r^{2} \cos (\tau z)}{2 f \sin (\tau z)}\right] J_{0}\left[\frac{k r \rho}{f \sin (\tau z)}\right] r \mathrm{~d} r,
\end{aligned}
$$

where $R$ is the radius of the field size in the input plane, and $r$ and $\rho$ are transverse coordinates in the input and output planes, respectively.

Table 4 shows the simulation results (obtained using the operator (12)) for circular autofocusing beams. The calculation parameters are the same as in the previous sections. In the case of circular symmetry, much more energy is directed to the focus; so, for the convenience of visualizing details, instead of the intensity, the amplitude is shown.

Note that the results for the circular tAi beam and the circular half-HG beam are very similar (Table 4, lines 1 and 2). Let us also consider the circular inverted LG beams:

$$
g_{i L G}(r)=\exp \left(-\frac{\left(r_{c}-r\right)^{2}}{2 \sigma^{2}}\right) L_{n}\left(\frac{\left(r_{c}-r\right)^{2}}{\sigma^{2}}\right),
$$

where $L_{n}(x)$ is the Laguerre polynomial of the $n$th order [33].

In this case, the autofocusing property is also observed, although the trajectory of the beams is somewhat different (Table 4 , line 3 ).

In papers $[2,4,12,34]$, autofocusing beams are considered in the form of sine functions with an arbitrary power-law dependence on the radius:

$$
g_{t S q}(r)=\sin \left[\left(\frac{r-r_{c}}{c}\right)^{q}\right] \operatorname{circ}\left(\frac{r}{b-a}\right),
$$

where $\operatorname{circ}(x)$ is the circular truncation function:

$$
\operatorname{circ}\left(\frac{r}{b-a}\right)=\left\{\begin{array}{l}
1, r \in[a, b] \\
0, \text { else }
\end{array}\right.
$$

Analytical expressions for the caustic trajectory of beams of Equation (14) were obtained earlier $[4,34,35]$ :

$$
\varsigma(z)=r_{c}+\frac{q-2}{q-1}\left[k^{-1} c^{-q}(q-1) z\right]^{-\frac{1}{q-2}} .
$$

As follows from Equation (16), the beam trajectory essentially depends on the value of the parameter $q$ (note, Equation (16) is applicable for $q \neq 0$ ). As seen in superlinear chirp beams $(q>2)$, the trajectory always has a hyperbolic form and an inverse dependence of the caustic radius on the distance $z$. For sublinear chirp beams $(1<q<2)$, the trajectory has a power-law dependence of the caustic radius on the distance $z$ (Equation (16) is rewritten for convenience):

$$
\varsigma(z)=r_{c}-\frac{2-q}{q-1}\left[k^{-1} c^{-q}(q-1) z\right]^{\frac{1}{2-q}}, \quad 1<q<2 .
$$

In this case, autofocusing is possible only when $r_{c} \neq 0$, and it occurs at the following distance:

$$
z_{\max }=\frac{k}{c^{-q}(q-1)^{q-1}} \cdot\left(\frac{r_{c}}{2-q}\right)^{2-q}, \quad 1<q<2
$$


Table 4. Comparison of circular autofocusing beams propagation.

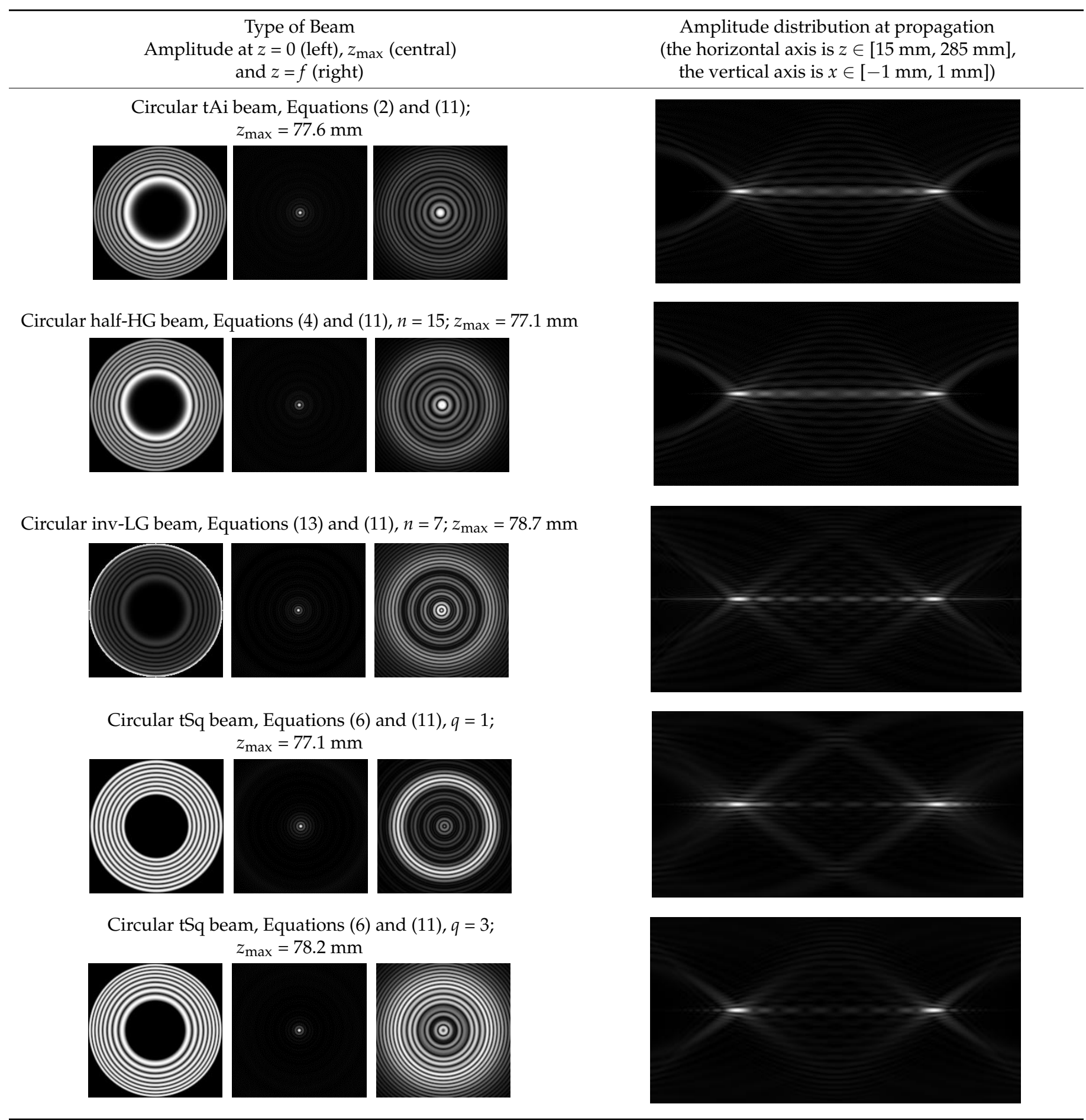

Similar to mirror beams, circular symmetry can be introduced in a different way so that instead of autofocusing, the beams diverge. These beams are called "ring" beams instead of "circular" beams. Examples of such beams are shown in Table 5. It is clearly seen that the LG mode completely retains its structure (up to scale) during propagation (Table 5, line 3). The ring tAi beam (Table 5, line 1) and the ring half-HG beam (Table 5, line 2) approximately retain their structures. The ring $\mathrm{tSq}$ beam with $q=1$ (Table 5, line 4) is actually a binary axicon that forms a Bessel beam in the near zone [36-38] and a double ring in the far zone or on the focal plane [39-41]. The ring tSq beam with $q=0.3$ (Table 5, 
line 5) corresponds to the binary fractional axicon [42-44], which provides the formation of a conical focal domain.

Table 5. Comparison of the diverging circular beams propagation.

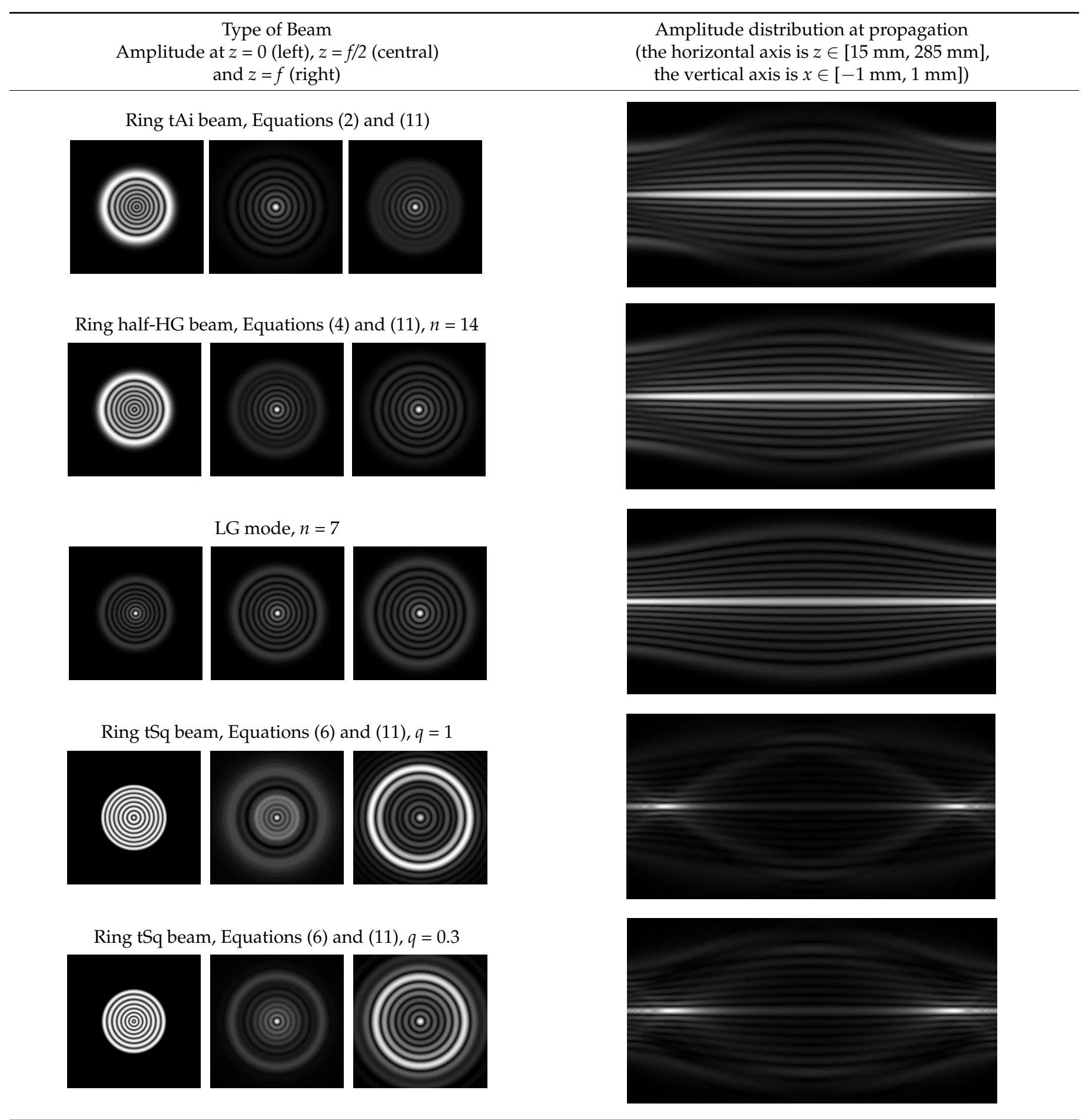

Thus, circular symmetry provides different beam properties depending on the nature of the radial function. For nonlinear functions, radial inversion and displacement significantly change the trajectory of the beam propagation. 


\section{Discussion}

Generation of abruptly autofocusing beams is based on a mirror or a circular symmetrization of accelerating beams with a nonlinear propagation trajectory, such as Airy and Pearcey beams, which are well-known from catastrophe theory [45-47]. It is also possible to form other beams with arbitrary trajectories or caustics [48-51].

This study showed that autofocusing beam generation is possible by symmetrization of fairly arbitrary distributions, for example, half-HG beams, truncated Bessel beams, and inverted LG beams, which were considered for the first time.

Truncated sinus functions with a power-law dependence on the argument are convenient for analytical calculations. In this case, for the power degree $q>2$ (superlinear chirp), the caustic trajectory has the form of a hyperbola, i.e., an inverse dependence of the caustic radius on the distance, and for $1<q<2$ (sublinear chirp), the trajectory has a power-law dependence of the caustic radius on the distance. It was shown in $[4,34,35]$ that superlinear chirp beams provide "sudden" and more abrupt autofocusing than sublinear chirp beams. This is due to the fact that at high values of the parameter $q$, the off-axis caustic retains the shape of a ring with approximately the same radius for a long time, and when it breaks off, a focal spot is abruptly and "unexpectedly" formed on the axis, which appears due to the axial caustic [52].

Note that autofocusing beams can also have a linear trajectory, for example, when a shifted Gaussian beam is symmetrized. An additional linear phase provides a simple and convenient way to control the autofocusing characteristics.

Various modifications of classic autofocusing beams are known. In particular, autofocusing beams acquire additional properties upon a vortex phase inserting [3,53-55]. A more complex modification provides segmentation and complication of the beam structure $[4,15,56,57]$, as well as rotation of a structured beam at propagation [58-60]. Another useful property of autofocusing beams is the possibility of forming optical bottles with complex shapes on their basis [61-63].

All these modifications can be applied to the beams considered in this study, which further expands the capabilities of structured beams in different applications.

\section{Conclusions}

A comparative numerical study of the propagation of various types of beams was carried out in this work that made it possible to draw the following main conclusions:

(1) The asymmetry and nonlinear dependence in the transverse distribution of beams provide nonlinearity to the propagation trajectory (acceleration property); however, for simple control of the linear trajectory of a conventional beam (for example, the Gaussian beam), one can use its displacement in the input plane and an additional linear phase function.

(2) The autofocusing property was obtained for a variety of beams (including half-HG beams, truncated Bessel beams, and inverted LG beams) when they were provided with mirror symmetry.

(3) A similar effect is seen for a circular symmetrization of asymmetric distributions, but the focus intensity, in this case, is much greater.

Thus, the formation of autofocusing beams is ensured by inserting a certain type of symmetry into fairly arbitrary distributions, which expands the capabilities of structured beams for a wide range of applications in optics and photonics, such as optical trapping and manipulation, laser structuring, and polarization conversion.

Funding: This research was funded by the Russian Foundation for Basic Research, grant number 20-07-00505.

Acknowledgments: This work was partly funded by the Ministry of Science and Higher Education within the government project of FSRC Crystallography and Photonics RAS, under agreement 007-GZ/Ch3363/26 (theoretical part). 
Conflicts of Interest: The author declares no conflict of interest.

\section{References}

1. Efremidis, N.K.; Christodoulides, D.N. Abruptly autofocusing waves. Opt. Lett. 2010, 35, 4045-4047. [CrossRef]

2. Chremmos, I.; Efremidis, N.K.; Christodoulides, D.N. Pre-engineered abruptly autofocusing beams. Opt. Lett. 2011, 36, 1890-1892. [CrossRef] [PubMed]

3. Davis, J.A.; Cottrell, D.M.; Sand, D. Abruptly autofocusing vortex beams. Opt. Express 2012, 20, 13302-13310. [CrossRef]

4. Porfirev, A.P.; Khonina, S.N. Generation of the azimuthally modulated circular superlinear Airy beams. J. Opt. Soc. Am. B 2017, 34, 2544-2549. [CrossRef]

5. Siviloglou, G.A.; Christodoulides, D.N. Accelerating finite energy Airy beams. Opt. Lett. 2007, 32, 979-981. [CrossRef] [PubMed]

6. Besieris, I.M.; Shaarawi, A.M. A note on an accelerating finite energy Airy beam. Opt. Lett. 2007, 32, 2447-2449. [CrossRef] [PubMed]

7. Zhang, P.; Prakash, J.; Zhang, Z.; Mills, M.S.; Efremidis, N.K.; Christodoulides, D.N.; Chen, Z. Trapping and guiding microparticles with morphing autofocusing Airy beams. Opt. Lett. 2011, 36, 2883-2885. [CrossRef]

8. Jiang, Y.; Huang, K.; Lu, X. Radiation force of abruptly autofocusing Airy beams on a Rayleigh particle. Opt. Express 2013, 21, 24413-24421. [CrossRef]

9. Manousidaki, M.; Papazoglou, D.G.; Farsari, M.; Tzortzakis, S. Abruptly autofocusing beams enable advanced multiscale photo-polymerization. Optica 2016, 3, 525-530. [CrossRef]

10. Panagiotopoulos, P.; Papazoglou, D.G.; Couairon, A.; Tzortzakis, S. Sharply autofocused ring-Airy beams transforming into non-linear intense light bullets. Nat. Commun. 2013, 4, 2622. [CrossRef]

11. Liu, S.; Wang, M.; Li, P.; Zhang, P.; Zhao, J. Abrupt polarization transition of vector autofocusing Airy beams. Opt. Lett. 2013, 38, 2416-2418. [CrossRef] [PubMed]

12. Degtyarev, S.A.; Volotovsky, S.G.; Khonina, S.N. Sublinearly chirped metalenses for forming abruptly autofocusing cylindrically polarized beams. J. Opt. Soc. Am. B 2018, 35, 1963-1969. [CrossRef]

13. Ring, J.; Lindberg, J.; Mourka, A.; Mazilu, M.; Dholakia, K.; Dennis, M. Auto-focusing and self-healing of Pearcey beams. Opt. Express 2012, 20, 18955-18966. [CrossRef] [PubMed]

14. Chen, X.; Deng, D.; Zhuang, J.; Yang, X.; Liu, H.; Wang, G. Nonparaxial propagation of abruptly autofocusing circular Pearcey Gaussian beams. Appl. Opt. 2018, 57, 8418-8423. [CrossRef] [PubMed]

15. Khonina, S.N.; Ustinov, A.V.; Porfirev, A.P. Aberration laser beams with autofocusing properties. Appl. Opt. 2018, 57, 1410-1416. [CrossRef]

16. Khonina, S.N. Specular and vortical Airy beams. Opt. Commun. 2011, 284, 4263-4271. [CrossRef]

17. Vaveliuk, P.; Lencina, A.; Rodrigo, J.A.; Matos, O.M. Symmetric Airy beams. Opt. Lett. 2014, 39, 2370-2373. [CrossRef]

18. Khonina, S.N.; Porfirev, A.P.; Fomchenkov, S.A.; Larkin, A.S.; Savelyev-Trofimov, A.B. Generation of closely located light spots using specular Airy laser beams. Comput. Opt. 2017, 41, 661-669. [CrossRef]

19. Belafhal, A.; Ez-Zariy, L.; Hennani, S.; Nebd, H. Theoretical introduction and generation method of a novel nondiffracting waves: Olver beams. Opt. Photon. J. 2015, 5, 234-246. [CrossRef]

20. Khonina, S.N.; Ustinov, A.V. Fractional Airy beams. J. Opt. Soc. Am. A 2017, 34, 1991-1999. [CrossRef]

21. Namias, V. The fractional Fourier transform and its application in quantum mechanics. JIMA 1980, 25, 241-265. [CrossRef]

22. Abe, S.; Sheridant, J.T. Generalization of the fractional Fourier transformation to an arbitrary linear lossless transformation: An operator approach. J. Phys. A Math. Gen. 1994, 27, 4179-4187. [CrossRef]

23. Alieva, T.; Bastiaans, M.J.; Calvo, M.L. Fractional transforms in optical information processing. EURASIP J. Appl. Signal Process. 2005, 10, 920687. [CrossRef]

24. Mendlovic, D.; Ozaktas, H.M. Fractional Fourier transformations and their optical implementation: I. J. Opt. Soc. Am. A 1993, 10, 1875-1881. [CrossRef]

25. Kirilenko, M.S.; Khonina, S.N. Formation of signals matched with vortex eigenfunctions of bounded double lens system. Opt. Commun. 2018, 410, 153-159. [CrossRef]

26. McMullin, J.N. The ABCD matrix in arbitrarily tapered quadratic-index waveguides. Appl. Opt. 1986, 25, 2184-2187. [CrossRef]

27. Mossoulina, O.A.; Kirilenko, M.S.; Khonina, S.N. Simulation of vortex laser beams propagation in parabolic index media based on fractional Fourier transform. J. Phys. Conf. Ser. 2016, 741, 012142. [CrossRef]

28. Berry, M.V.; Balazs, N.L. Nonspreading wave packets. Am. J. Phys. 1979, 47, 264. [CrossRef]

29. Saari, P. Laterally accelerating Airy pulses. Opt. Express 2008, 16, 10303-10308. [CrossRef]

30. Li, H.H.; Wang, J.G.; Tang, M.M.; Li, X.Z. Propagation properties of cosh-Airy beams. J. Mod. Opt. 2018, 65, 314-320. [CrossRef]

31. Kovalev, A.A.; Kotlyar, V.V.; Zaskanov, S.G. Structurally stable three-dimensional and two-dimensional laser half Pearcey beams. Comput. Opt. 2014, 38, 193-197. [CrossRef]

32. Feng, Z.; Yan, W.; Lu, L. Dual self-accelerating properties of one-dimensional finite energy Pearcey beam. Results Phys. 2019, 15, 102656. [CrossRef]

33. Abramowitz, M.; Stegun, I.A. Handbook of Mathematical Functions; Dover: New York, NY, USA, 1972.

34. Khonina, S.N.; Porfirev, A.P.; Ustinov, A.V. Sudden autofocusing of superlinear chirp beams. J. Opt. 2018, 20, 025605. [CrossRef] 
35. Ustinov, A.V.; Khonina, S.N. Properties of off-axis caustics of autofocusing chirp beams. Comput. Opt. 2020, 44, $721-727$. [CrossRef]

36. Turunen, J.; Vasara, A.; Friberg, A.T. Holographic generation of diffraction-free beams. Appl. Opt. 1988, 27, 3959-3962. [CrossRef] [PubMed]

37. Akturk, S.; Arnold, C.L.; Prade, B.; Mysyrowicz, A. Generation of high quality tunable Bessel beams using a liquid-immersion axicon. Opt. Commun. 2009, 282, 3206-3209. [CrossRef]

38. Khonina, S.N.; Serafimovich, P.G.; Savelyev, D.A.; Pustovoi, I.A. Diffraction at binary microaxicons in the near field. J. Opt. Technol. 2012, 79, 626-631. [CrossRef]

39. Fedotowsky, A.; Lehovec, K. Far field diffraction patterns of circular gratings. Appl. Opt. 1974, 13, 2638-2642. [CrossRef]

40. Amidror, I. Fourier spectrum of radially periodic images. J. Opt. Soc. Am. A 1997, 14, 816-826. [CrossRef]

41. Khonina, S.N.; Porfirev, A.P. 3D transformations of light fields in the focal region implemented by diffractive axicons. Appl. Phys. B 2018, 124, 191. [CrossRef]

42. Davidson, N.; Friesem, A.A.; Hasman, E. Holographic axilens: High resolution and long focal depth. Opt. Lett. 1991, 16, 523-525. [CrossRef]

43. Khonina, S.N.; Ustinov, A.V. Very compact focal spot in the near-field of the fractional axicon. Opt. Commun. 2017, 391, 24-29. [CrossRef]

44. Gorelick, S.; Paganin, D.M.; de Marco, A. Axilenses: Refractive micro-optical elements with arbitrary exponential profiles. APL Photon. 2020, 5, 106110. [CrossRef]

45. Poston, T.; Stewart, I. Catastrophe Theory and Its Applications; Dover Publication Inc.: Mineola, NY, USA, 1978; ISBN 0-486-69271-X.

46. Gilmore, R. Catastrophe Theory for Scientists and Engineers; Dover: New York, NY, USA, 1993; ISBN 978-0-486-67539-8.

47. Kharitonov, S.I.; Volotovsky, S.G.; Khonina, S.N.; Kazanskiy, N.L. Diffraction catastrophes and asymptotic analysis of caustics from axisymmetric optical elements. Proc. SPIE 2019, 11146, 111460K. [CrossRef]

48. Greenfield, E.; Segev, M.; Walasik, W.; Raz, O. Accelerating light beams along arbitrary convex trajectories. Phys. Rev. Lett. 2011, 106, 213902. [CrossRef]

49. Froehly, L.; Courvoisier, F.; Mathis, A.; Jacquot, M.; Furfaro, L.; Giust, R.; Lacourt, P.A.; Dudley, J.M. Arbitrary accelerating micron-scale caustic beams in two and three dimensions. Opt. Express 2011, 19, 16455-16465. [CrossRef]

50. Chremmos, I.D.; Chen, Z.; Christodoulides, D.N.; Efremidis, N.K. Abruptly autofocusing and autodefocusing optical beams with arbitrary caustics. Phys. Rev. A 2012, 85, 023828. [CrossRef]

51. Zhao, Z.; Xie, C.; Ni, D.; Zhang, Y.; Li, Y.; Courvoisier, F.; Hu, M. Scaling the abruptly autofocusing beams in the direct-space. Opt. Express 2017, 25, 30598-30605. [CrossRef] [PubMed]

52. Soifer, V.A.; Kharitonov, S.I.; Khonina, S.N.; Volotovsky, S.G. Caustics of vortex optical beams. Dokl. Phys. 2019, 64, 276-279. [CrossRef]

53. Chen, B.; Chen, C.; Peng, X.; Peng, Y.; Zhou, M.; Deng, D. Propagation of sharply autofocused ring Airy Gaussian vortex beams. Opt. Express 2015, 23, 19288-19298. [CrossRef] [PubMed]

54. Jiang, Y.; Zhao, S.; Yu, W.; Zhu, X. Abruptly autofocusing property of circular Airy vortex beams with different initial launch angles. J. Opt. Soc. Am. A 2018, 35, 890-894. [CrossRef]

55. Sun, C.; Deng, D.; Wang, G.; Yang, X.; Hong, W. Abruptly autofocusing properties of radially polarized circle Pearcey vortex beams. Opt. Commun. 2020, 457, 124690. [CrossRef]

56. Zhang, Y.; Li, P.; Liu, S.; Han, L.; Cheng, H.; Zhao, J. Manipulating spin-dependent splitting of vector abruptly autofocusing beam by encoding cosine-azimuthal variant phases. Opt. Express 2016, 24, 28409-28418. [CrossRef]

57. Yan, X.; Guo, L.; Cheng, M.; Chai, S. Free-space propagation of autofocusing Airy vortex beams with controllable intensity gradients. Chin. Opt. Lett. 2019, 17, 040101. [CrossRef]

58. Brimis, A.; Makris, K.G.; Papazoglou, D.G. Tornado waves. Opt. Lett. 2020, 45, 280-283. [CrossRef]

59. Wu, Y.; Xu, C.; Lin, Z.; Qiu, H.; Fu, X.; Chen, K.; Deng, D. Abruptly autofocusing polycyclic tornado ring Airy beam. New J. Phys. 2020, 22, 093045. [CrossRef]

60. Khonina, S.N.; Porfirev, A.P.; Ustinov, A.V.; Butt, M.A. Generation of complex transverse energy flow distributions with autofocusing optical vortex beams. Micromachines 2021, 12, 297. [CrossRef]

61. Chremmos, I.D.; Zhang, P.; Prakash, J.; Efreidis, N.K.; Christodoulides, D.N.; Chen, Z.G. Fourier-space generation of abruptly autofocusing beams and optical bottle beams. Opt. Lett. 2011, 36, 3675-3677. [CrossRef]

62. Xu, C.J.; Wu, Y.; Deng, D.M. Multioptical bottles from the second order chirped symmetric airy vortex beams. Opt. Lett. 2020, 45, 3502-3505. [CrossRef]

63. Wu, Y.; Lin, Z.J.; Xu, C.J.; Fu, X.M.; Chen, K.H.; Qiu, H.X.; Deng, D.M. Off-axis and multi optical bottles from the astigmatic phase ring airy gaussian vortex beams in the free space. Ann. Der Phys. 2020, 532, 2000188. [CrossRef] 